\title{
BMJ Open Prognostic value of derived neutrophil- to-lymphocyte ratio (dNLR) in patients with non-small cell lung cancer receiving immune checkpoint inhibitors: a meta-analysis
}

\author{
Tao Yang (D) , ${ }^{1,2}$ Lizheng Hao, ${ }^{1}$ Xinyu Yang, ${ }^{1}$ Changyong Luo, ${ }^{3}$ Guomi Wang, ${ }^{3}$ \\ Caroline Lin Cai, ${ }^{4}$ Shuo Qi (D) , ${ }^{5}$ Zhong Li ${ }^{6}$
}

To cite: Yang T, Hao L, Yang $\mathrm{X}$, et al. Prognostic value of derived neutrophilto-lymphocyte ratio (dNLR) in patients with non-small cell lung cancer receiving immune checkpoint inhibitors: a meta-analysis. BMJ Open 2021;11:e049123. doi:10.1136/ bmjopen-2021-049123

- Prepublication history and additional online supplemental material for this paper are available online. To view these files, please visit the journal online (http://dx.doi.org/10. 1136/bmjopen-2021-049123)

TY and LH contributed equally.

Received 16 January 2021 Accepted 19 June 2021

Check for updates

(C) Author(s) (or their employer(s)) 2021. Re-use permitted under CC BY-NC. No commercial re-use. See rights and permissions. Published by BMJ.

For numbered affiliations see end of article.

Correspondence to

Professor Zhong Li;

a2916@bucm.edu.cn and

Dr Shuo Qi;

shuoqi@bucm.edu.cn

\section{ABSTRACT}

Objectives Derived neutrophil-to-lymphocytes ratio (dNLR) has recently been reported as a novel potential biomarker associated with prognosis of non-small cell lung cancer (NSCLC). However, evidence for the prognostic utility of dNLR in patients with NSCLC treated with immune checkpoint inhibitors (ICls) remains inconsistent. The objective of this work was to evaluate the association between pretreatment dNLR and prognosis of patients with NSCLC treated with ICls.

Design This study followed the Preferred Reporting Items for Systematic Reviews and Meta-Analyses guidelines.

Data sources PubMed, EMBASE, Web of Science and the Cochrane Library were searched for eligible studies up to 16 October 2020.

Eligibility criteria (1) Human subjects receiving ICls therapy and who had been diagnosed with NSCLC; (2) the baseline values of dNLR were obtained; (3) the objective of the study was to investigate the relationships between $\mathrm{dNLR}$ and overall survival (OS) or progression-free survival (PFS) in NSCLC and (4) HR and 95\% Cl were displayed in the original article or could be extracted from KaplanMeier curves.

Data extraction and synthesis Two investigators extracted data independently. Data synthesis was performed via systematic review and meta-analysis of eligible cohort studies. Meta-analysis was performed with Cochran's $Q$ test and $I^{2}$ statistics. Publication bias of studies was assessed by Begg's test and Egger's test. We used V.12.0 of the Stata statistical software.

Results This analysis included eight studies (2456 cases) on the prognostic utility of dNLR in ICI therapy for NSCLC. The results indicate that higher dNLR significantly predicted poor $\mathrm{OS}(\mathrm{HR}=1.65,95 \% \mathrm{Cl} 1.46$ to $1.88 ; p<0.001)$ and PFS $(\mathrm{HR}=1.38,95 \% \mathrm{Cl} 1.23$ to $1.55 ; p<0.001)$. Subgroup analyses of OS-related studies indicated that there were similar results in stratifications by ethnicity, sample size, type of HR and dNLR cut-off value. As for PFS-related studies, subgroup analyses showed no significant difference in Asian populations. Publication biases were not detected using Begg's test and Egger's linear regression test.
Strengths and limitations of this study

- This is the first study to evaluate the prognostic value of pretreatment derived neutrophil-tolymphocyte ratio in patients with non-small cell lung cancer (NSCLC) who treated with immune checkpoint inhibitors (ICls).

- This meta-analysis may provide novel prognostic guidance for patients with NSCLC treated with ICls.

- All the studies included in this meta-analysis were retrospective cohort studies, and the number of eligible studies was $<10$, so there may be some retrospective bias and publication bias.

Conclusions This meta-analysis indicated that elevated pretreatment dNLR may be a negative prognostic predictor for patients with NSCLC treated with ICls. More largesample and higher-quality studies are warranted to support our findings.

PROSPERO registration number CRD42021214034.

\section{INTRODUCTION}

Worldwide, lung cancer remains the leading cause of cancer death, with an estimated 2.2 million new cases and 1.8 million deaths in 2020. ${ }^{1}$ Non-small cell lung cancer (NSCLC) accounts for about $85 \%$ of primary lung cancers and includes three main pathological types: squamous cell carcinoma, adenocarcinoma and large cell lung cancer. ${ }^{2}$ The treatment strategy for NSCLC depends on the stage of the cancer. Early-stage patients should be treated with surgical resection, while advanced-stage patients are mainly treated with systematic therapy. The 5-year survival rates for NSCLC range from $14 \%$ to $49 \%$ for stage I-IIIA patients, and are less than $5 \%$ for stage IIIB-IV disease. ${ }^{3}$ In the past 10 years, the application of immune checkpoint inhibitors (ICIs) in the treatment of NSCLC 


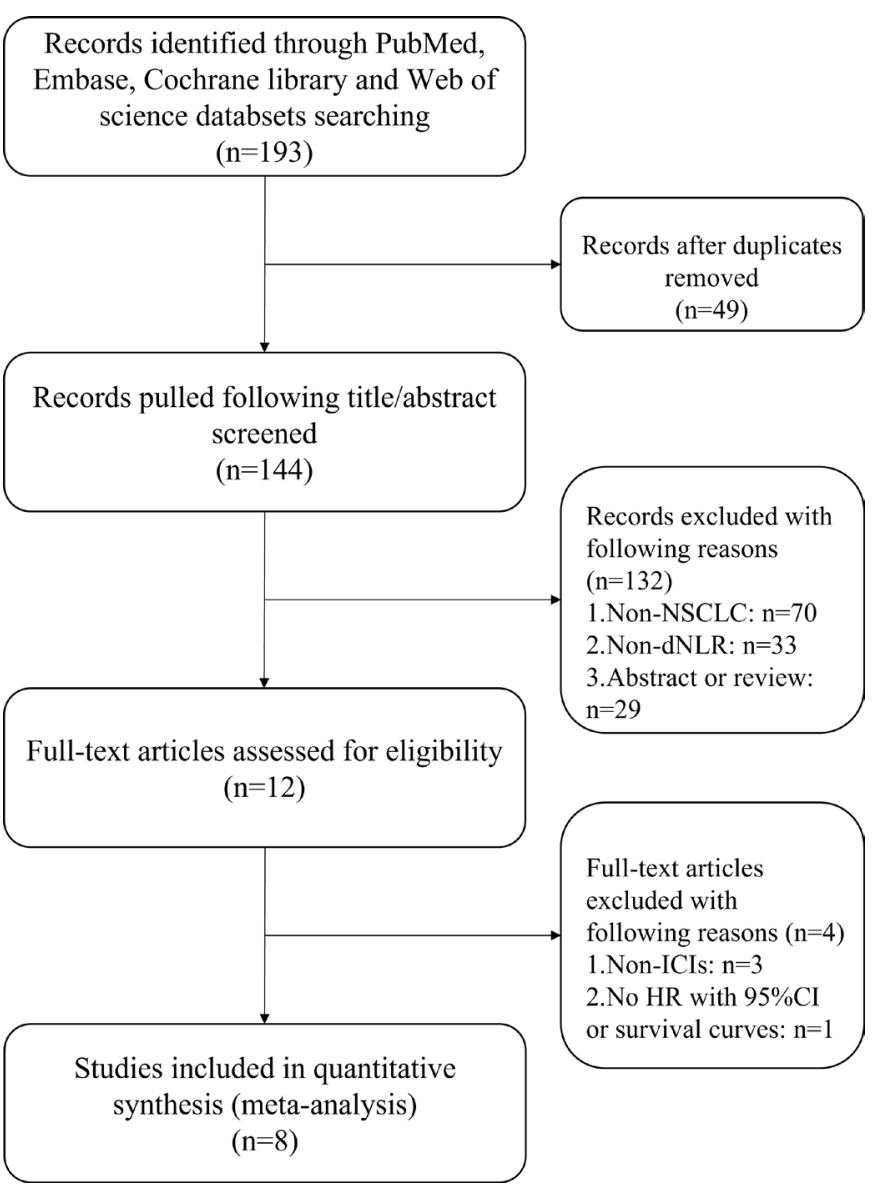

Figure 1 Flow chart of the eligible studies. dNLR, derived neutrophil-to-lymphocyte ratio; ICls, immune checkpoint inhibitors; NSCLC, non-small cell lung cancer.

has improved the therapeutic landscape for this intractable disease. PD-1 and programmed cell death-ligand 1 (PD-L1) inhibitors have shown encouraging results in NSCLC (pembrolizumab and nivolumab, eg) and they have been approved by the US Food and Drug Administration for the treatment of advanced NSCLC. ${ }^{45}$ The latest phase 3 study showed that nivolumab was demonstrated a superior overall survival (OS) versus docetaxel at 2 years in NSCLC. ${ }^{6}$ And a real-life cohort of patients with advanced NSCLC treated with pembrolizumab demonstrated similar progression-free survival (PFS) to the pivotal clinical trial. ${ }^{7}$ Some patients with advanced NSCLC have shown OS or PFS benefits from ICI treatment after chemoradiotherapy. ${ }^{89}$

Despite significant clinical improvements, not all ICI treatments are effective in patients with NSCLC. Some valuable biomarkers that predict ICI response, such as PD-L1, tumour mutational burden and tumourinfiltrating lymphocytes which could indicate the status of the tumour immune microenvironment have led to more effective application of ICIs. ${ }^{10}$ However, most of these biomarkers are detected in an invasive manner, which depends heavily on sufficient tumour tissue. Thus, there is an urgent need to explore and evaluate better biomarkers for selecting patients suitable for ICI treatment.
Inflammation processes have been proven to be mechanisms of immune resistance in patients with cancer which can promote tumour growth and invasion and activate carcinogenic signalling pathways. ${ }^{11}$ In the clinical practice, peripheral serum indicators are used to evaluate systemic inflammation, and some of them are associated with prognosis and therapeutic response of patients with cancer. ${ }^{12} 13$ The common haematological inflammatory indicators include white blood cells (WBC), lymphocytes and $\mathrm{C}$ reactive protein (CRP). Derived neutrophil-tolymphocyte ratio (dNLR) is a novel potential biomarker for systemic inflammation, which can be calculated by absolute value of neutrophils and value of leucocyte count. ${ }^{14}$ DNLR has been used to assess response to immunotherapy in various cancers, including NSCLC. ${ }^{15-17}$ Recent studies showed the predictive utility of pretreatment dNLR in urological cancer and breast cancer. ${ }^{18} 19$ However, evidence of the association between the prognosis of NSCLC and dNLR remains mixed. Therefore, the objective of our study was to explore the relationship between pretreatment dNLR and survival in patients with NSCLC treated with ICIs.

\section{METHODS}

\section{Patient and public involvement}

It was not appropriate or possible to involve patients or the public in the design, conduct, reporting, or dissemination plans of our research.

\section{Design}

This study followed the Preferred Reporting Items for Systematic Reviews and Meta-Analyses guidelines (online supplemental file 1).

\section{Search strategy and study inclusion}

Our meta-analysis was conducted to explore the association between dNLR and prognosis of patients with NSCLC treated with ICIs. We conducted a search of four electronic journal databases: PubMed, EMBASE, Web of Science and the Cochrane Library. The search consisted of three parts: (1) the subject words (Emtree in EMBASE and MeSH in other databases) and free words of NSCLC were searched respectively, (2) the abbreviations and specific names of ICIs were searched, (3) dNLR and its full name were also searched. The last search was updated on 16 October 2020 (online supplemental file 2).

The inclusion criteria were as follows: (1) human subjects receiving ICIs therapy and who had been diagnosed with NSCLC; $(2)$ the baseline values of dNLR were obtained; (3) the objective of the study was to investigate the relationships between dNLR and OS or PFS in NSCLC; (4) HR and 95\% CI were displayed in the original article or could be extracted from Kaplan-Meier curves.

The exclusion criteria were as follows: (1) studies including subjects with other diseases; (2) case reports, reviews, meta-analyses, conference abstracts and letters; 


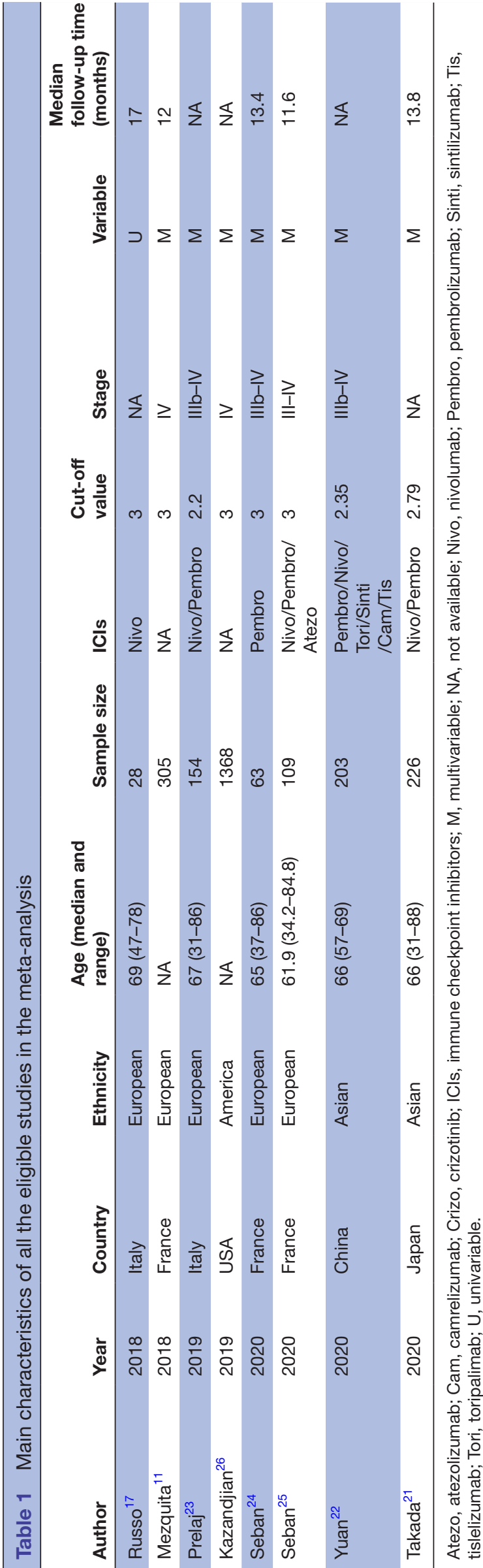

(3) duplicate publications and (4) we were unable to acquire the full text or data from the text.

\section{Quality assessment}

We evaluated the quality of the included studies using the Newcastle-Ottawa Scale (NOS), ${ }^{20}$ which assesses three aspects of the studies: selection, comparability and outcome. Each study could be given a maximum of nine stars. A higher number of stars indicated better study quality.

\section{Data extraction}

Two investigators independently extracted data. Any disagreement was settled by discussion until agreement was reached or by consulting a third investigator. Data extracted were author, year of publication, study districts, age, sample size, type of ICIs, median follow-up time, cutoff value of dNLR and clinical stage. As for quantitative data, HRs with 95\% CI of OS and PFS were also acquired from the included studies.

\section{Statistical analysis}

To evaluate the association between pretreatment dNLR and survival outcomes of the patients with NSCLC treated with ICIs, HRs with 95\% CI were gathered to give the effective value. We assessed the heterogeneity of the eligible studies by using Cochran's Q test and $\mathrm{I}^{2}$ statistics. $\mathrm{I}^{2}>50 \%$ and $\mathrm{p}<0.05$ in the Cochran's $\mathrm{Q}$ test were considered to indicate significant heterogeneity, and the random effects model was applied to calculate the pooled HRs. If heterogeneity was not significant, the fixed effects model was used. Subgroup analysis was conducted to assess heterogeneity among the results of different studies and explore the stability of results in different stratifications. Publication bias of studies was assessed by Begg's test and Egger's test. All $p$ values were two sided, and $p<0.05$ was considered statistically significant. STATA statistical software V.12.0 was used for all statistical analysis in this study.

\section{RESULTS}

\section{Study characteristics}

A total of 193 articles were retrieved using the initial search strategies. After multiple screening processes, 8 studies with a total of 2456 patients, published between 2018 and 2020 , were finally included in our meta-analysis. The flow chart of study inclusion is shown in figure 1. Among all studies, participants in two studies were Asian ${ }^{21} 22$ and in the other six were European or American. ${ }^{11} 1723-26$ HRs and $95 \%$ CIs were reported exactly in seven studies, ${ }^{11}$ 21-26 while the remaining study ${ }^{17}$ reported only HR and $p$ value; we then estimated 95\% CI for that study based on HR and $\mathrm{p}$ value. ${ }^{27}$ The calculation formula is as follows:

$$
\begin{aligned}
& \mathrm{SE}=\frac{\log (H R)}{-0.862+\sqrt{2.404 \times \log (P)}} \\
& \text { Lower } 95 \%=e^{\log (H R)-1.96 \times S E} \\
& \text { Upper } 95 \%=e^{\log (H R)+1.96 \times S E}
\end{aligned}
$$



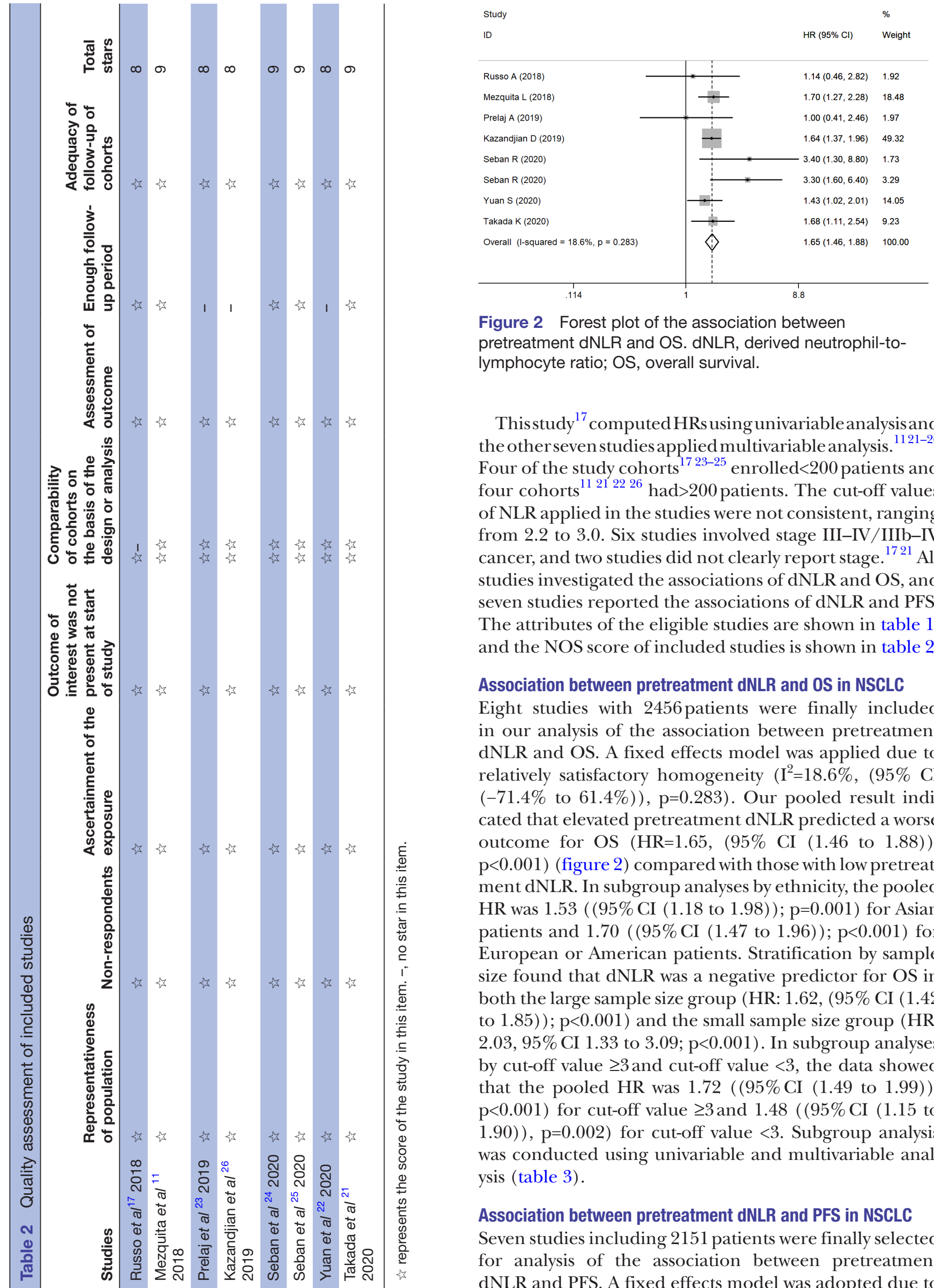

Figure 2 Forest plot of the association between pretreatment dNLR and OS. dNLR, derived neutrophil-tolymphocyte ratio; OS, overall survival.

This study ${ }^{17}$ computed HRs using univariable analysis and the other seven studies applied multivariable analysis. ${ }^{1121-26}$ Four of the study cohorts ${ }^{172-25}$ enrolled $<200$ patients and four cohorts ${ }^{11} 212226$ had $>200$ patients. The cut-off values of NLR applied in the studies were not consistent, ranging from 2.2 to 3.0. Six studies involved stage III-IV/IIIb-IV cancer, and two studies did not clearly report stage. ${ }^{172}$ All studies investigated the associations of dNLR and OS, and seven studies reported the associations of $d N L R$ and PFS. The attributes of the eligible studies are shown in table 1 , and the NOS score of included studies is shown in table 2.

\section{Association between pretreatment dNLR and OS in NSCLC}

Eight studies with 2456 patients were finally included in our analysis of the association between pretreatment dNLR and OS. A fixed effects model was applied due to relatively satisfactory homogeneity $\left(\mathrm{I}^{2}=18.6 \%\right.$, (95\% CI $(-71.4 \%$ to $61.4 \%)), \mathrm{p}=0.283)$. Our pooled result indicated that elevated pretreatment dNLR predicted a worse outcome for OS (HR=1.65, (95\% CI (1.46 to 1.88)); $\mathrm{p}<0.001$ ) (figure 2) compared with those with low pretreatment dNLR. In subgroup analyses by ethnicity, the pooled HR was 1.53 ( $(95 \%$ CI (1.18 to 1.98)); $\mathrm{p}=0.001)$ for Asian patients and 1.70 ( $(95 \%$ CI $(1.47$ to 1.96$))$; $\mathrm{p}<0.001)$ for European or American patients. Stratification by sample size found that dNLR was a negative predictor for OS in both the large sample size group (HR: 1.62, (95\% CI (1.42 to 1.85$)$ ); $\mathrm{p}<0.001$ ) and the small sample size group (HR: $2.03,95 \%$ CI 1.33 to $3.09 ; \mathrm{p}<0.001)$. In subgroup analyses by cut-off value $\geq 3$ and cut-off value $<3$, the data showed that the pooled HR was 1.72 ( $(95 \%$ CI (1.49 to 1.99$)$ ), $\mathrm{p}<0.001)$ for cut-off value $\geq 3$ and 1.48 ( $(95 \%$ CI $(1.15$ to $1.90)$ ), $\mathrm{p}=0.002)$ for cut-off value $<3$. Subgroup analysis was conducted using univariable and multivariable analysis (table 3 ).

Association between pretreatment dNLR and PFS in NSCLC

Seven studies including 2151 patients were finally selected for analysis of the association between pretreatment dNLR and PFS. A fixed effects model was adopted due to 


\section{Study}

ID

HR $(95 \% \mathrm{Cl}) \quad$ Weight

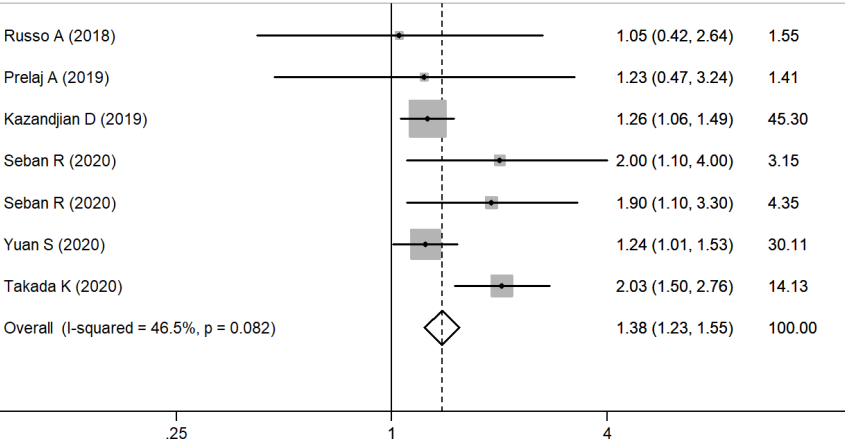

Figure 3 Forest plot of the association between pretreatment dNLR and PFS. dNLR, derived neutrophil-tolymphocyte ratio; PFS, progression-free survival.

$\mathrm{I}^{2}=46.5 \%(95 \%$ CI $(-27.0 \%$ to $77.4 \%))$ and $\mathrm{p}=0.082$. The results demonstrated that high pretreatment dNLR was significantly associated with poorer PFS (HR $=1.38,(95 \%$ CI (1.23 to 1.55)); $\mathrm{p}<0.001$ ) (figure 3 ) compared with low pretreatment dNLR. Subgroup analysis was performed by ethnicity; the results showed that dNLR was a negative predictor for NSCLC in European or American patients $(\mathrm{HR}=1.33$, (95\% CI (1.14 to 1.55)); $\mathrm{p}<0.001)$, but in Asian $\mathrm{dNLR}$ and PFS have no significant relationship $(\mathrm{HR}=1.57$, ( $95 \%$ CI ( 0.97 to 2.54$)$ ); $\mathrm{p}=0.068$ ). In the small sample size group, the pooled HR was 1.67 ( $(95 \%$ CI (1.17 to 2.37$))$; $\mathrm{p}=0.005)$, and in the large sample size group the HR was 1.43 ((95\% CI (1.10 to 1.85$)) ; \mathrm{p}=0.007)$. Subgroup analyses by cut-off value of dNLR showed that the pooled HR was 1.33 ( $(95 \%$ CI $(1.14$ to 1.55$)), \mathrm{p}<0.001)$ for cutoff value $\geq 3$ and 1.51 ( $(95 \%$ CI $(1.01$ to 2.26$)), p=0.043)$ for cut-off value $<3$. Furthermore, subgroup analysis was conducted using univariable and multivariable analysis, and the results also illustrated the interrelation between baseline dNLR and PFS (table 3 ).

\section{Publication bias}

We conducted Begg's and Egger's linear regression test to assess publication bias. OS publication bias was not discovered in studies with dNLR ( $\operatorname{Pr}>|\mathrm{z}|=0.902$ for Begg's test and $\mathrm{P}>|\mathrm{t}|=0.648$ for Egger's test); publication bias was also not detected for PFS (Pr $>|\mathrm{z}|=0.764$ and $\mathrm{P}>|\mathrm{t}|=0.392$, respectively). The plots of Begg's test and Egger's test are shown in figure 4 .

\section{DISCUSSION}

This meta-analysis evaluated the results of 2456 patients with NSCLC in eight studies. The results showed that high level dNLR was a significant predictor of worse OS $(\mathrm{HR}=1.65$, (95\% CI $(1.46$ to 1.88$)) ; \mathrm{p}<0.001)$ and PFS $(\mathrm{HR}=1.38$, (95\% CI $(1.23$ to 1.55$)) ; \mathrm{p}<0.001)$ of patients with NSCLC treated with ICIs. Subgroup analyses of OS-related studies indicated similar results in stratifications by ethnicity, sample size, type of HR and dNLR cut-off value. In PFS-related studies, subgroup analyses 
A

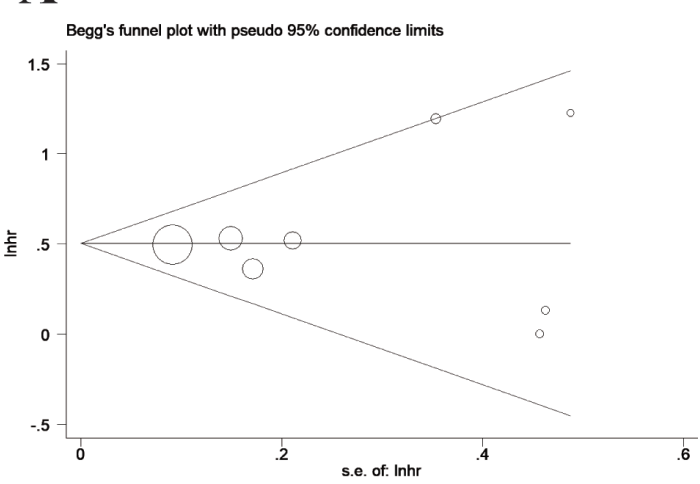

C

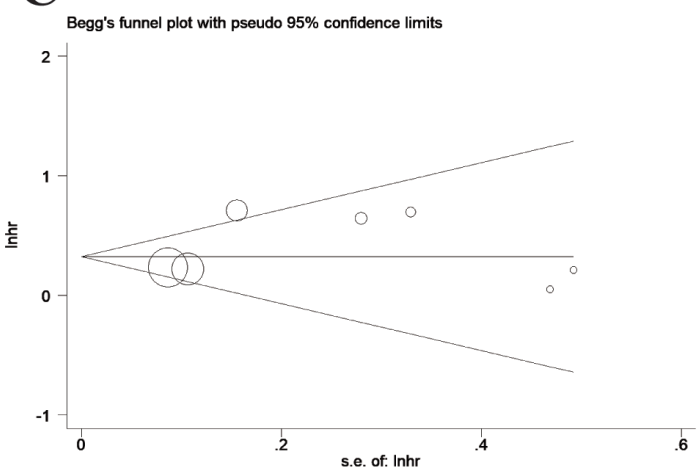

B

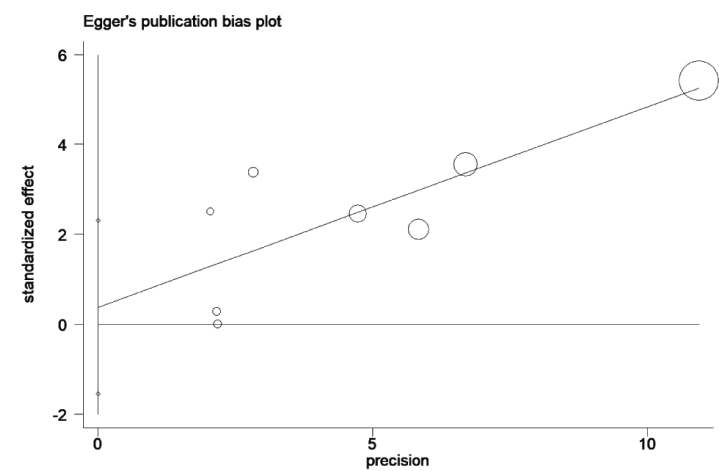

D

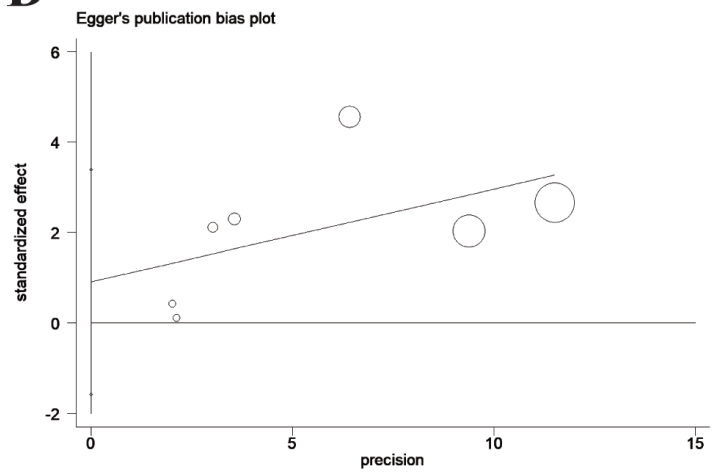

Figure 4 Funnel plot for analysis of publication bias. (A) Funnel plot established using Begg's test for studies with overall survival (OS) ; (B) funnel plot using Egger's test for studies with OS. (C) Funnel plot established utilising Begg's test for studies with progression-free survival (PFS); (D) funnel plot using Egger's test for studies with PFS.

showed that there was no significant difference in the Asian sample group, but Asian sample subgroup only included two studies, which might weaken the credibility of the results of subgroup analysis. We conclude that pretreatment dNLR may be an important biomarker of the prognosis of patients with NSCLC treated with ICIs.

Inflammation tends to lead to the development of cancer and stimulates all stages of tumourigenesis through multiple mechanisms. ${ }^{28}$ Induction of inflammation can bring increased mutagenesis, leading to collection of mutations in normal tissue that can further cause tumour formation. ${ }^{29}{ }^{30}$ Unlike in earlier stages of oncogenesis, cancer-related inflammation plays a crucial role in regulation of metastasis and leads to worse mortality. ${ }^{31}$ Additionally, the inflammation process has been suggested as a reason for immune resistance in cancer patients. The cellular effectors of inflammation are significant elements of the tumour microenvironment that break down adaptive immune responses and impede responses to antitumour agents. ${ }^{32}$ Moreover, a peripheral proinflammatory condition has been linked to poor prognosis in patients with cancer. ${ }^{11}$ Many routine blood indices including WBC, CRP, absolute neutrophil count and lactate dehydrogenase level have been evaluated as potential inflammatory biomarkers, which are associated with worse survival in various types of cancer. ${ }^{33-35}$ Novel biomarkers such as NLR, lymphocyte-monocyte ratio and lymphocyte-platelet ratio have also been used to assess inflammatory status in several cancer types, including NSCLCs. ${ }^{36-38}$ In particular, NLR is a well-studied prognostic predictor in patients with NSCLC, and some metaanalyses have confirmed the predictive value of NLR in patients with NSCLC. ${ }^{39} 40$

Recent studies indicated that dNLR is a novel serum marker of inflammatory in patients with NSCLC treated with ICIs. ${ }^{17}$ Al Although some studies have suggested relationships between NLR and survival and therapeutic outcomes in patients with NSCLC treated with anti-PD-1 inhibitors, ${ }^{12} 4243$ dNLR may be more strongly linked because it includes monocytes and other granulocytes. Immature or poorly differentiated neutrophils can be released in a proinflammatory environment, which increases neutrophil generation rapidly. dNLR seems to reflect this negative inflammation more comprehensively. Our study demonstrated that dNLR may be a valuable prognostic serum biomarker for clinicians' decision making in NSCLC ICIs treatment. Future studies should pay more attentions to the prognostic effect of dNLR on the patients with NSCLC with ICIs. A larger sample study is needed to verify our results.

In our study, most included studies have chosen a dNLR cut-off value of 3 to distinguish the prognosis of patients with NSCLC treated with ICIs, however, the selection and source of dNLR cut-off values were rarely mentioned in original studies. We performed subgroup analysis according to different dNLR cut-off levels, the 
results show that significant $\mathrm{HR}$ of OS and PFS could be produced by all subgroups. It is also probably necessary to use receiver operating characteristic curves or other tools to determine the optimal pretreatment dNLR cutoff value based on large sample data, so that dNLR can be better applied to clinical practice. In addition, the included original studies did not provide information that might affect dNLR, such as related diseases, previous treatment, etc. These factors may lead to differences in the baseline characteristics of the patients, which may influence the interpretation of our results. In the future study, we will pay more attention to this aspect, and more comprehensive original studies should be used to obtain more reliable results.

Several limitations of our meta-analysis require careful consideration. First, the eligible studies were all retrospective, retrospective study is more prone to several bias including selection, recall and measurement biases, so these retrospective biases may influence the accuracy of results. Although recall bias was not explicit mentioned in the original studies, the systematic error between the accuracy or integrity and the real situation is often the result of the memory distortion or incomplete recall of the research object when collecting the information. In addition, most of the included studies were retrospective and single institution case series, and as mentioned above, the original study did not provide more information such as other diseases and previous treatment and so on, which may lead to selection bias. Second, although neither Begg's test nor Egger's test showed publication bias in this study, the effectiveness of the two tests was low when the number of meta-analyses was $<10$. In addition, our study mainly searched English-language databases. Hence, publication bias should also be considered.

In conclusion, this meta-analysis revealed that elevated pretreatment dNLR may be a negative prognostic index for patients with NSCLC treated with ICIs. Future welldesigned and large-scale studies are needed to validate the result.

\section{Author affiliations}

${ }^{1}$ Beijing University of Chinese Medicine Affiliated Dongzhimen Hospital, Beijing, China

${ }^{2}$ Beijing Shijitan Hospital, Capital Medical University, Beijing, People's Republic of China

${ }^{3}$ Beijing University of Chinese Medicine, Beijing, China

${ }^{4}$ London College of Chinese Medicine, London, UK

${ }^{5}$ Department of Thyroid, Sun Simiao hospital, Beijing University of Chinese

Medicine, Tongchuan, China

${ }^{6}$ Department of Hematology and Oncology, Beijing University of Chinese Medicine Affiliated Dongzhimen Hospital, Beijing, China

Contributors ZL and SQ put forward the idea of research. The search strategy was developed and conducted by TY, LH and XY. LH and XY independently screened the titles and abstracts of all included studies. Data extraction was performed by LH, $\mathrm{XY}, \mathrm{CL}$ and GW. TY and LH conducted the meta-analysis. Manuscript was written by TY and CLC.

Funding The authors have not declared a specific grant for this research from any funding agency in the public, commercial or not-for-profit sectors.

Competing interests None declared.
Patient consent for publication Not required.

Provenance and peer review Not commissioned; externally peer reviewed.

Data availability statement Data are available in a public, open access repository. No additional data available.

Supplemental material This content has been supplied by the author(s). It has not been vetted by BMJ Publishing Group Limited (BMJ) and may not have been peer-reviewed. Any opinions or recommendations discussed are solely those of the author(s) and are not endorsed by BMJ. BMJ disclaims all liability and responsibility arising from any reliance placed on the content. Where the content includes any translated material, BMJ does not warrant the accuracy and reliability of the translations (including but not limited to local regulations, clinical guidelines, terminology, drug names and drug dosages), and is not responsible for any error and/or omissions arising from translation and adaptation or otherwise.

Open access This is an open access article distributed in accordance with the Creative Commons Attribution Non Commercial (CC BY-NC 4.0) license, which permits others to distribute, remix, adapt, build upon this work non-commercially, and license their derivative works on different terms, provided the original work is properly cited, appropriate credit is given, any changes made indicated, and the use is non-commercial. See: http://creativecommons.org/licenses/by-nc/4.0/.

ORCID iDs

Tao Yang http://orcid.org/0000-0003-3127-3944

Shuo Qi http://orcid.org/0000-0001-7559-4606

\section{REFERENCES}

1 Sung H, Ferlay J, Siegel RL, et al. Global cancer statistics 2020: GLOBOCAN estimates of incidence and mortality worldwide for 36 cancers in 185 countries. CA Cancer J Clin 2021;71:209-49.

2 Duma N, Santana-Davila R, Molina JR. Non-Small cell lung cancer: epidemiology, screening, diagnosis, and treatment. Mayo Clin Proc 2019;94:1623-40.

$3 \mathrm{Ko} \mathrm{EC}$, Raben D, Formenti SC. The integration of radiotherapy with immunotherapy for the treatment of non-small cell lung cancer. Clin Cancer Res 2018;24:5792-806.

4 Vokes EE, Ready N, Felip E, et al. Nivolumab versus docetaxel in previously treated advanced non-small-cell lung cancer (CheckMate 017 and CheckMate 057): 3-year update and outcomes in patients with liver metastases. Ann Oncol 2018;29:959-65.

5 Herbst RS, Baas P, Kim D-W, et al. Pembrolizumab versus docetaxel for previously treated, PD-L1-positive, advanced non-small-cell lung cancer (KEYNOTE-010): a randomised controlled trial. Lancet 2016;387:1540-50.

6 Lu S, Wang J, Cheng Y, et al. Nivolumab versus docetaxel in a predominantly Chinese patient population with previously treated advanced non-small cell lung cancer: 2-year follow-up from a randomized, open-label, phase 3 study (CheckMate 078). Lung Cancer 2021:152:7-14.

7 Amrane K, Geier M, Corre R, et al. First-line pembrolizumab for non-small cell lung cancer patients with PD-L1 $\geq 50 \%$ in a multicenter real-life cohort: The PEMBREIZH study. Cancer Med 2020;9:2309-16.

8 Antonia SJ, Villegas A, Daniel D, et al. Durvalumab after chemoradiotherapy in stage III Non-Small-Cell lung cancer. N Engl J Med Overseas Ed 2017;377:1919-29.

9 Antonia SJ, Villegas A, Daniel D, et al. Overall survival with Durvalumab after chemoradiotherapy in stage III NSCLC. N Engl J Med 2018;379:2342-50.

10 Bodor JN, Boumber Y, Borghaei H. Biomarkers for immune checkpoint inhibition in non-small cell lung cancer (NSCLC). Cancer 2020;126:260-70.

11 Mezquita L, Auclin E, Ferrara R, et al. Association of the lung immune prognostic index with immune checkpoint inhibitor outcomes in patients with advanced non-small cell lung cancer. JAMA Oncol 2018;4:351-7.

12 Bagley SJ, Kothari S, Aggarwal C, et al. Pretreatment neutrophilto-lymphocyte ratio as a marker of outcomes in nivolumab-treated patients with advanced non-small-cell lung cancer. Lung Cancer 2017;106:1-7.

13 Pine JK, Morris E, Hutchins GG, et al. Systemic neutrophil-tolymphocyte ratio in colorectal cancer: the relationship to patient survival, tumour biology and local lymphocytic response to tumour. Br J Cancer 2015;113:204-11.

14 Proctor MJ, McMillan DC, Morrison DS, et al. A derived neutrophil to lymphocyte ratio predicts survival in patients with cancer. $\mathrm{Br} J$ Cancer 2012;107:695-9. 
15 Afzal MZ, Sarwar T, Shirai K. Prognostic significance of hematological indices in malignant melanoma treated with immune checkpoint inhibitors. J Immunother 2019;42:251-64.

16 Bruixola G, Caballero J, Papaccio F, et al. Prognostic nutritional index as an independent prognostic factor in locoregionally advanced squamous cell head and neck cancer. ESMO Open 2018;3:e000425.

17 Russo A, Franchina T, Ricciardi GRR, et al. Baseline neutrophilia derived neutrophil-to-lymphocyte ratio (dNLR), platelet-tolymphocyte ratio (PLR), and outcome in non small cell lung cancer (NSCLC) treated with nivolumab or docetaxel. J Cell Physiol 2018;233:6337-43

18 Su S, Liu L, Li C, et al. Prognostic role of pretreatment derived neutrophil to lymphocyte ratio in urological cancers: a systematic review and meta-analysis. Int J Surg 2019;72:146-53.

19 Duan J, Pan L, Yang M. Preoperative elevated neutrophil-tolymphocyte ratio (NLR) and derived NLR are associated with poor prognosis in patients with breast cancer: a meta-analysis. Medicine 2018;97:e13340.

20 . The Newcastle-Ottawa scale (NOS) for assessing the quality of nonrandomised studies in meta-analyses. Symposium on Systematic Reviews: Beyond the Basics, 2014.

21 Takada K, Takamori S, Yoneshima Y, et al. Serum markers associated with treatment response and survival in non-small cell lung cancer patients treated with anti-PD-1 therapy. Lung Cancer 2020;145:18-26.

22 Yuan S, Xia Y, Shen L, et al. Development of nomograms to predict therapeutic response and prognosis of non-small cell lung cancer patients treated with anti-PD-1 antibody. Cancer Immunol Immunother 2021;70:533-46.

23 Prelaj A, Rebuzzi SE, Pizzutilo P, et al. Epsilon: a prognostic score using clinical and blood biomarkers in advanced non-smallcell lung cancer treated with immunotherapy. Clin Lung Cancer 2020;21:365-77.

24 Seban R-D, Assié J-B, Giroux-Leprieur E, et al. Association of the metabolic score using baseline FDG-PET/CT and dNLR with immunotherapy outcomes in advanced NSCLC patients treated with first-line pembrolizumab. Cancers 2020;12:2234-14.

25 Seban R-D, Mezquita L, Berenbaum A, et al. Baseline metabolic tumor burden on FDG PET/CT scans predicts outcome in advanced NSCLC patients treated with immune checkpoint inhibitors. Eur $J$ Nucl Med Mol Imaging 2020;47:1147-57.

26 Kazandjian D, Gong Y, Keegan P, et al. Prognostic value of the lung immune prognostic index for patients treated for metastatic nonsmall cell lung cancer. JAMA Oncol 2019;5:1481-5.

27 Altman DG, Bland JM. How to obtain the confidence interval from a $P$ value. BMJ 2011;343:d2090.

28 Greten FR, Grivennikov SI. Inflammation and cancer: triggers, mechanisms, and consequences. Immunity 2019;51:27-41.
29 Canli Özge, Nicolas AM, Gupta J, et al. Myeloid cell-derived reactive oxygen species induce epithelial mutagenesis. Cancer Cell 2017;32:869-83.

30 Robles Al, Traverso G, Zhang M, et al. Whole-Exome sequencing analyses of inflammatory bowel disease-associated colorectal cancers. Gastroenterology 2016;150:931-43.

31 Rothwell PM, Wilson M, Price JF, et al. Effect of daily aspirin on risk of cancer metastasis: a study of incident cancers during randomised controlled trials. Lancet 2012;379:1591-601.

32 Mantovani A, Allavena P, Sica A, et al. Cancer-Related inflammation. Nature 2008;454:436-44.

33 Soyano AE, Dholaria B, Marin-Acevedo JA, et al. Peripheral blood biomarkers correlate with outcomes in advanced non-small cell lung cancer patients treated with anti-PD-1 antibodies. J Immunother Cancer 2018:6:129.

34 Wang $\mathrm{H}$, Wang M-S, Zhou Y-H, et al. Prognostic values of LDH and CRP in cervical cancer. Onco Targets Ther 2020;13:1255-63.

35 Yoon Cl, Park S, Cha YJ, et al. Associations between absolute neutrophil count and lymphocyte-predominant breast cancer. Breast 2020;50:141-8

36 Xiao X, Wang S, Long G. C-Reactive protein is a significant predictor of improved survival in patients with advanced non-small cell lung cancer. Medicine 2019;98:e16238.

37 Machado D, Marques C, Dias M, et al. Inflammatory prognostic biomarkers in advanced non-small cell lung cancer. Pulmonology 2019;25:181-3.

38 Cedrés S, Torrejon D, Martínez A, et al. Neutrophil to lymphocyte ratio (NLR) as an indicator of poor prognosis in stage IV non-small cell lung cancer. Clin Transl Oncol 2012;14:864-9.

39 Wang Z, Zhan P, Lv Y, et al. Prognostic role of pretreatment neutrophil-to-lymphocyte ratio in non-small cell lung cancer patients treated with systemic therapy: a meta-analysis. Trans/ Lung Cancer Res 2019;8:214-26.

40 Sacdalan DB, Lucero JA, Sacdalan DL. Prognostic utility of baseline neutrophil-to-lymphocyte ratio in patients receiving immune checkpoint inhibitors: a review and meta-analysis. Onco Targets Ther 2018;11:955-65.

41 Blanc-Durand F, Auclin E, Planchard D, et al. Association of lung immune prognostic index (LIPI) with survival of first line immune checkpoint inhibitors single agent or in combination with chemotherapy in untreated advanced NSCLC patients. Ann Oncol 2019;30:xi5.

42 Park W, Lopes G. Perspectives: neutrophil-to-lymphocyte ratio as a potential biomarker in immune checkpoint inhibitor for non-small-cel lung cancer. Clin Lung Cancer 2019;20:143-7.

43 Jiang T, Bai Y, Zhou F, et al. Clinical value of neutrophil-tolymphocyte ratio in patients with non-small-cell lung cancer treated with PD-1/PD-L1 inhibitors. Lung Cancer 2019;130:76-83. 